\title{
Rural and Urban Children Have Lower Rates of Health Insurance Coverage and are More Often Covered by Public Plans
}

\author{
MARYBETH J. MATTINGLY AND MICHELLE L. STRANSKY
}

$\Lambda$ s legislators continue to debate an overhaul of America's health insurance system, it is critical to remember America's children. In order to better understand children's current access to health care, we show estimates of health insurance coverage for children under age 18 (Table 1) and public and private insurance (Table 2) by region, state, and place in 2008 from recently released American Community Survey data. ${ }^{1}$

The differences highlighted reveal the crucial role public health insurance plans play in providing for the needs of children, particularly those residing in rural and urban places. Yet nearly 10 percent of our youngest citizens are still without health care coverage. As major changes to America's health insurance system are considered, the needs of the youngest citizens should be at the forefront.

\section{Data}

This analysis is based upon U.S. Census Bureau estimates from the 2008 American Community Survey. ${ }^{3}$ Tables were produced by aggregating information from detailed tables available on American FactFinder (http://factfinder.census. gov/home/saff/main.html?_lang=en). These estimates are based on survey data, thus caution must be used in making comparisons. Confidence intervals for rural estimates were created according to the 2008 American Community Survey Accuracy of the Data ${ }^{4}$; confidence intervals for non-rural places were calculated using the margins of error for those with health insurance and the population under 18 , as recommended by Census Bureau officials. If the confidence intervals overlap, differences are not statistically significant $(\mathrm{p}<0.05) .{ }^{5}$ Differences discussed in the text are statistically significant.

\section{Key Findings}

- Nearly one in ten American children has no health insurance coverage.

- Rural children have lower rates of health insurance coverage in every region except the South, where children residing in central cities have the lowest rate of coverage.

- In the following fourteen states, rural children have the lowest rate of health insurance coverage, lower than both suburban places and central cities: Alaska, Arizona, California, Colorado, Florida, Hawaii, Indiana, Louisiana, Minnesota, Montana, New York, Oregon, South Dakota, and Wisconsin. ${ }^{2}$

- In the following eight states, rural children have rates similar to children in central cities but significantly lower than children in the suburbs: Alabama, Arkansas, lowa, Kansas, Missouri, Ohio, Pennsylvania, and Texas.

- Of those children who have health insurance, an estimated 28 percent rely on a public option.

- Of children with health insurance, almost two-thirds are covered by private health insurance.

- Across the United States, a significantly smaller share of children in rural places and in central cities is covered by private health insurance than in the suburbs. A larger percent of children in rural places and central cities is covered by public plans than in the suburbs.

- In the South and the West, rural children have the lowest rates of private health insurance coverage compared to their central city and suburban counterparts. Children living in central cities have less private health insurance coverage than children in rural and suburban places in the Northeast and the Midwest. 
Table 1. Health insurance among children by place size ${ }^{1}$ in 2008

2008 AMERICAN COMMUNITY SURVEY²

\begin{tabular}{|c|c|c|c|c|c|c|c|c|c|c|c|c|c|c|c|c|}
\hline & \multicolumn{4}{|c|}{ TOTAL } & \multicolumn{4}{|c|}{ RURAL } & \multicolumn{4}{|c|}{ SUBURBAN } & \multicolumn{4}{|c|}{ CENTRAL CITY } \\
\hline & \multirow{3}{*}{$\begin{array}{c}\text { Population } \\
\text { under } 18\end{array}$} & \multicolumn{3}{|c|}{ Percent insured } & \multirow{3}{*}{$\begin{array}{l}\text { Population } \\
\text { under } 18\end{array}$} & \multicolumn{3}{|c|}{ Percent insured } & \multirow{3}{*}{$\begin{array}{c}\text { Population } \\
\text { under } 18\end{array}$} & \multicolumn{3}{|c|}{ Percent insured } & & & cent ins & red \\
\hline & & $\%$ & $95^{\circ}$ & & & $\%$ & $95^{\circ}$ & & & $\%$ & $95^{\circ}$ & & Population & $\%$ & $95^{\circ}$ & ${ }_{6}^{6} \mathrm{Cl}$ \\
\hline & & & Lower & Upper & & & Lower & Upper & & & Lower & Upper & & & Lower & Upper \\
\hline United States & $73,786,055$ & 90.1 & 90.0 & 90.2 & $11,392,125$ & 89.1 & 89.0 & 89.2 & $38,729,045$ & 91.1 & 91.0 & 91.3 & $23,664,885$ & 88.8 & 88.6 & 88.9 \\
\hline Northeast & $12,365,293$ & 94.4 & 94.2 & 94.6 & $1,098,288$ & 92.4 & 91.9 & 92.9 & $7,510,146$ & 95.0 & 94.8 & 95.3 & $3,756,859$ & 93.7 & 93.5 & 94.0 \\
\hline Midwest & $16,035,365$ & 93.4 & 93.2 & 93.5 & $3,504,092$ & 91.6 & 91.3 & 91.8 & $8,085,156$ & 94.7 & 94.5 & 95.0 & $4,446,117$ & 92.3 & 92.1 & 92.5 \\
\hline South & $27,478,112$ & 87.5 & 87.4 & 87.7 & $5,149,260$ & 88.2 & 87.9 & 88.4 & $14,221,964$ & 88.4 & 88.1 & 88.6 & $8,106,888$ & 85.7 & 85.4 & 86.1 \\
\hline West & $17,907,285$ & 88.0 & 87.8 & 88.2 & $1,640,485$ & 84.7 & 84.3 & 85.0 & $8,911,779$ & 89.1 & 88.7 & 89.4 & $7,355,021$ & 87.4 & 87.0 & 87.9 \\
\hline Alabama & $1,119,732$ & 92.0 & 91.2 & 92.7 & 310,775 & 91.2 & 90.2 & 92.1 & 521,758 & 93.1 & 92.9 & 93.3 & 287,199 & 90.8 & 89.1 & 92.5 \\
\hline Alaska & 180,431 & 87.1 & 85.5 & 88.8 & 45,196 & 78.6 & 78.0 & 79.3 & 41,883 & 91.2 & 87.2 & 95.1 & 80,264 & 89.2 & 86.1 & 92.4 \\
\hline Arizona & $1,705,841$ & 83.8 & 82.9 & 84.7 & 126,060 & 79.1 & 77.5 & 80.7 & 737,825 & 86.4 & 84.8 & 88.0 & 841,956 & 82.3 & 81.0 & 83.5 \\
\hline Arkansas & 701,489 & 91.3 & 90.3 & 92.2 & 265,556 & 91.0 & 90.0 & 91.9 & 232,263 & 93.6 & 93.3 & 93.9 & 203,670 & 89.0 & 87.4 & 90.6 \\
\hline California & $9,347,714$ & 89.2 & 88.9 & 89.5 & 167,602 & 85.3 & 83.9 & 86.7 & $4,796,101$ & 89.6 & 89.2 & 90.1 & $4,384,011$ & 88.9 & 88.2 & 89.5 \\
\hline Colorado & $1,203,936$ & 85.6 & 84.7 & 86.6 & 150,251 & 77.3 & 76.1 & 78.5 & 612,614 & 89.2 & 87.9 & 90.5 & 441,071 & 83.5 & 81.7 & 85.4 \\
\hline Connecticut & 809,967 & 95.1 & 94.5 & 95.7 & 65,559 & 95.3 & 93.2 & 97.3 & 520,643 & 96.2 & 95.4 & 97.0 & 223,765 & 92.6 & 91.2 & 93.9 \\
\hline Delaware & 206,043 & 92.2 & 90.4 & 94.1 & 40,652 & 88.5 & 85.5 & 91.6 & 141,942 & 93.3 & 91.0 & 95.5 & 23,449 & 92.5 & 87.1 & 98.0 \\
\hline Washington, DC & 111,263 & 95.7 & 93.8 & 97.7 & $\mathrm{~N} / \mathrm{A}$ & $N / A$ & N/A & N/A & N/A & N/A & N/A & N/A & 111,263 & 95.7 & 93.8 & 97.7 \\
\hline Florida & $3,996,332$ & 82.5 & 81.8 & 83.1 & 211,748 & 80.5 & 80.4 & 80.7 & $2,777,608$ & 82.6 & 81.8 & 83.4 & $1,006,976$ & 82.5 & 81.3 & 83.6 \\
\hline Georgia & $2,536,058$ & 88.5 & 87.9 & 89.2 & 440,550 & 88.6 & 87.9 & 89.4 & $1,699,242$ & 88.7 & 87.8 & 89.5 & 396,266 & 87.8 & 87.0 & 88.6 \\
\hline Hawaii & 284,493 & 96.4 & 95.3 & 97.5 & 85,335 & 92.6 & 89.8 & 95.4 & 136,076 & 97.9 & 97.0 & 98.8 & 63,082 & 98.1 & 96.3 & 99.9 \\
\hline Idaho & 412,970 & 86.3 & 84.8 & 87.9 & 138,708 & 83.5 & 82.4 & 84.6 & 152,380 & 87.0 & 84.5 & 89.6 & 121,882 & 88.7 & 87.8 & 89.6 \\
\hline Illinois & $3,175,518$ & 94.4 & 94.0 & 94.8 & 355,189 & 94.8 & 94.0 & 95.6 & $1,755,878$ & 95.1 & 94.8 & 95.4 & $1,064,451$ & 93.0 & 92.2 & 93.8 \\
\hline Indiana & $1,579,871$ & 89.8 & 89.0 & 90.7 & 338,311 & 85.6 & 84.4 & 86.7 & 741,442 & 92.3 & 91.3 & 93.2 & 500,118 & 89.2 & 87.7 & 90.6 \\
\hline lowa & 708,251 & 94.4 & 93.7 & 95.1 & 295,944 & 93.8 & 92.9 & 94.6 & 206,871 & 96.4 & 95.2 & 97.6 & 205,436 & 93.3 & 91.8 & 94.9 \\
\hline Kansas & 698,223 & 91.7 & 90.9 & 92.6 & 240,575 & 89.9 & 88.9 & 91.0 & 232,351 & 94.7 & 94.1 & 95.3 & 225,297 & 90.6 & 88.8 & 92.5 \\
\hline Kentucky & $1,004,446$ & 93.0 & 92.3 & 93.7 & 406,875 & 92.0 & 91.2 & 92.8 & 362,971 & 93.4 & 92.3 & 94.4 & 234,600 & 94.3 & 92.9 & 95.7 \\
\hline Louisiana & $1,105,606$ & 92.3 & 91.5 & 93.0 & 291,396 & 89.7 & 88.6 & 90.7 & 495,847 & 92.8 & 92.1 & 93.5 & 318,363 & 93.8 & 92.0 & 95.6 \\
\hline Maine & 274,570 & 92.6 & 91.2 & 94.0 & 109,825 & 91.6 & 90.2 & 93.0 & 126,972 & 94.3 & 92.2 & 96.4 & 37,773 & 90.1 & 86.3 & 93.9 \\
\hline Maryland & $1,338,996$ & 94.7 & 94.2 & 95.3 & 67,084 & 93.4 & 91.5 & 95.2 & $1,041,838$ & 95.2 & 94.6 & 95.7 & 230,074 & 93.3 & 91.9 & 94.7 \\
\hline Massachusetts & $1,426,668$ & 97.9 & 97.6 & 98.2 & N/A & $N / A$ & $\mathrm{~N} / \mathrm{A}$ & $\mathrm{N} / \mathrm{A}$ & $1,095,357$ & 97.9 & 97.5 & 98.4 & 325,978 & 97.9 & 97.6 & 98.2 \\
\hline Michigan & $2,388,510$ & 94.8 & 94.5 & 95.2 & 399,683 & 94.8 & 94.3 & 95.3 & $1,344,583$ & 95.3 & 95.0 & 95.7 & 644,244 & 93.7 & 93.0 & 94.5 \\
\hline Minnesota & $1,248,041$ & 93.7 & 93.3 & 94.1 & 310,107 & 91.7 & 90.9 & 92.4 & 667,573 & 94.8 & 94.2 & 95.4 & 270,361 & 93.3 & 92.5 & 94.1 \\
\hline Mississippi & 762,887 & 87.3 & 86.1 & 88.6 & 419,401 & 87.0 & 86.4 & 87.6 & 254,013 & 89.3 & 87.6 & 91.0 & 89,473 & 83.2 & 80.0 & 86.4 \\
\hline Missouri & $1,418,342$ & 92.9 & 92.4 & 93.3 & 363,927 & 91.7 & 91.1 & 92.3 & 768,847 & 93.9 & 93.4 & 94.5 & 285,568 & 91.4 & 90.0 & 92.9 \\
\hline Montana & 221,829 & 85.5 & 83.7 & 87.3 & 143,339 & 82.3 & 81.7 & 83.0 & 28,729 & 93.7 & 90.5 & 96.8 & 49,761 & 90.0 & 86.6 & 93.4 \\
\hline Nebraska & 444,803 & 93.1 & 92.3 & 93.9 & 172,938 & 91.4 & 90.4 & 92.3 & 114,233 & 96.0 & 94.8 & 97.2 & 157,632 & 93.0 & 91.1 & 94.9 \\
\hline Nevada & 666,937 & 79.8 & 78.3 & 81.3 & 46,649 & 81.4 & 80.6 & 82.1 & 322,197 & 81.3 & 80.9 & 81.6 & 283,500 & 78.2 & 76.3 & 80.1 \\
\hline New Hampshire & 292,592 & 94.9 & 93.9 & 95.9 & 90,589 & 92.3 & 90.8 & 93.9 & 148,373 & 95.4 & 93.9 & 96.8 & 44,573 & 98.1 & 96.7 & 99.5 \\
\hline New Jersey & $2,045,334$ & 92.8 & 92.3 & 93.3 & $\mathrm{~N} / \mathrm{A}$ & $N / A$ & N/A & N/A & $1,814,274$ & 93.2 & 92.7 & 93.7 & 231,060 & 89.4 & 87.9 & 90.9 \\
\hline New Mexico & 500,994 & 86.3 & 85.2 & 87.5 & 173,161 & 85.5 & 84.3 & 86.7 & 152,951 & 85.5 & 83.7 & 87.3 & 174,882 & 87.8 & 85.8 & 89.8 \\
\hline New York & $4,402,183$ & 94.2 & 93.9 & 94.5 & 322,254 & 90.7 & 89.7 & 91.7 & $1,871,678$ & 94.9 & 94.4 & 95.4 & $2,208,251$ & 94.2 & 93.7 & 94.6 \\
\hline North Carolina & $2,237,294$ & 90.2 & 89.6 & 90.7 & 636,993 & 90.0 & 89.3 & 90.7 & 923,588 & 90.4 & 89.7 & 91.1 & 676,713 & 90.1 & 89.1 & 91.0 \\
\hline North Dakota & 140,900 & 92.3 & 90.6 & 93.9 & 71,693 & 92.0 & 90.6 & 93.5 & 28,964 & 93.9 & 89.7 & 98.1 & 40,243 & 91.5 & 87.6 & 95.4 \\
\hline Ohio & $2,727,548$ & 92.8 & 92.3 & 93.3 & 523,564 & 90.8 & 89.9 & 91.6 & $1,567,974$ & 94.3 & 93.8 & 94.7 & 636,010 & 90.7 & 89.8 & 91.7 \\
\hline Oklahoma & 903,224 & 87.1 & 86.3 & 87.9 & 311,193 & 85.2 & 84.4 & 86.0 & 329,228 & 88.6 & 86.8 & 90.4 & 262,803 & 87.4 & 85.9 & 88.9 \\
\hline Oregon & 865,115 & 87.2 & 86.1 & 88.4 & 183,427 & 84.2 & 82.6 & 85.7 & 395,085 & 87.4 & 85.9 & 89.0 & 286,603 & 89.0 & 87.4 & 90.5 \\
\hline Pennsylvania & $2,757,063$ & 93.9 & 93.4 & 94.4 & 413,096 & 92.8 & 91.9 & 93.7 & $1,741,008$ & 94.8 & 94.4 & 95.3 & 602,959 & 91.9 & 90.7 & 93.0 \\
\hline Rhode Island & 228,072 & 94.3 & 93.2 & 95.5 & $\mathrm{~N} / \mathrm{A}$ & $\mathrm{N} / \mathrm{A}$ & $\mathrm{N} / \mathrm{A}$ & N/A & 155,170 & 95.1 & 94.0 & 96.2 & 72,902 & 92.7 & 89.6 & 95.7 \\
\hline South Carolina & $1,064,430$ & 87.4 & 86.6 & 88.3 & 249,473 & 87.6 & 86.6 & 88.5 & 658,577 & 87.4 & 86.2 & 88.7 & 156,380 & 87.3 & 84.4 & 90.2 \\
\hline South Dakota & 196,191 & 91.6 & 90.5 & 92.7 & 102,855 & 88.0 & 86.9 & 89.2 & 43,330 & 95.0 & 93.8 & 96.2 & 50,006 & 95.9 & 94.2 & 97.7 \\
\hline Tennessee & 1,476,111 & 92.8 & 92.2 & 93.4 & 374,058 & 93.0 & 92.3 & 93.7 & 591,618 & 94.2 & 93.4 & 94.9 & 510,435 & 91.1 & 89.8 & 92.3 \\
\hline Texas & $6,714,046$ & 82.2 & 81.8 & 82.6 & 727,847 & 81.0 & 80.8 & 81.1 & $2,913,466$ & 84.5 & 83.9 & 85.1 & $3,072,733$ & 80.3 & 79.6 & 81.0 \\
\hline Utah & 849,122 & 86.9 & 85.8 & 88.0 & 88,872 & 87.1 & 86.9 & 87.3 & 593,371 & 88.6 & 87.4 & 89.8 & 166,879 & 80.6 & 76.9 & 84.3 \\
\hline Vermont & 128,844 & 96.0 & 95.0 & 97.0 & 82,575 & 94.8 & 93.5 & 96.1 & 36,671 & 97.7 & 97.2 & 98.3 & $\mathrm{~N} / \mathrm{A}$ & $\mathrm{N} / \mathrm{A}$ & N/A & $\mathrm{N} / \mathrm{A}$ \\
\hline Virginia & $1,815,340$ & 92.4 & 91.9 & 92.9 & 225,516 & 90.7 & 90.0 & 91.4 & $1,107,964$ & 92.9 & 92.3 & 93.4 & 481,860 & 92.1 & 90.7 & 93.4 \\
\hline Washington & $1,540,488$ & 91.5 & 90.9 & 92.2 & 176,722 & 92.0 & 90.9 & 93.1 & 928,781 & 91.4 & 90.4 & 92.5 & 434,985 & 91.5 & 90.2 & 92.9 \\
\hline West Virginia & 384,815 & 93.3 & 92.2 & 94.4 & 170,143 & 91.6 & 90.3 & 92.9 & 170,041 & 95.2 & 93.6 & 96.7 & 44,631 & 92.8 & 89.7 & 95.8 \\
\hline Wisconsin & $1,309,167$ & 94.8 & 94.3 & 95.3 & 329,306 & 91.9 & 91.1 & 92.7 & 613,110 & 96.5 & 95.8 & 97.3 & 366,751 & 94.6 & 93.8 & 95.4 \\
\hline Wyoming & 127,415 & 91.3 & 89.3 & 93.2 & 87,484 & 90.3 & 89.0 & 91.7 & N/A & N/A & N/A & N/A & 26,145 & 94.1 & 91.3 & 96.9 \\
\hline Puerto Rico & 980,770 & 94.5 & 93.9 & 95.1 & 38,435 & 95.5 & 94.8 & 96.2 & 706,500 & 94.9 & 94.2 & 95.7 & 219,308 & 93.0 & 91.3 & 94.8 \\
\hline
\end{tabular}

'Levels of urbanization are defined as follows: Rural consists of American Community Survey geographic components "not in metropolitan or micropolitan statistical area" and "in micropolitan statistical area"; suburban includes "in metropolitan statistical area-not in principal city" and central city includes "in metropolitan statistical area-in principal city."

${ }^{2}$ Data are based on 2008 American Community Survey estimates.

Source: American Community Survey 
Table 2. Private and Public Health Insurance among Children by Place Size ${ }^{1}$ in 2008

2008 AMERICAN COMMUNITY SURVEY²

\begin{tabular}{|c|c|c|c|c|c|c|c|c|c|c|c|c|c|c|c|c|c|c|c|c|c|c|c|c|}
\hline & \multicolumn{6}{|c|}{ TOTAL } & \multicolumn{6}{|c|}{ RURAL } & \multicolumn{6}{|c|}{ SUBURBAN } & \multicolumn{6}{|c|}{ CENTRAL CITY } \\
\hline & \multicolumn{3}{|c|}{$\begin{array}{l}\text { Percent Private } \\
\text { Insurance }\end{array}$} & \multicolumn{3}{|c|}{$\begin{array}{l}\text { Percent Public } \\
\text { Insurance }\end{array}$} & & & & & & & & & & & & & & & & & & \\
\hline & $\%$ & & & $\%$ & & & $\%$ & & & $\%$ & & & $\%$ & & & $\%$ & & & $\%$ & & & $\%$ & & \\
\hline & & ower & pper & & Lower & Upper & & $\mathrm{bW}$ & pper & & Lou & Upper & & on & Upper & & ow & $\mathrm{Jpp}$ & & or & Upper & & _ow & Jppe \\
\hline itec & 4.1 & 63.9 & 64.3 & 28.3 & 28.2 & 28.5 & 57.7 & 57.5 & 57.9 & 34.9 & 34.6 & 35.3 & 71.2 & 71.0 & 71.4 & 22.0 & 21.8 & 22.1 & 55.6 & 55.4 & 55.9 & 35.6 & 35.3 & 35.9 \\
\hline orth & 0.7 & 0.3 & 71.0 & 6.7 & 26.3 & 27.1 & 4.9 & 64.6 & 65.3 & 1.6 & 30.7 & 32.6 & 9.6 & 79.2 & 80.0 & 17.8 & 17.4 & 18.1 & 54.5 & 53.7 & 55.3 & 3.1 & 42.4 & 43.9 \\
\hline idwest & 69.5 & 69.1 & 69.9 & 6.7 & 26.4 & 27.0 & 65.9 & 65.7 & 66.1 & 29.8 & 29.2 & 30.3 & 8.0 & 77.6 & 78.4 & 19.1 & 18.8 & 19.5 & 7.0 & 56.4 & 57.6 & 38.1 & 37.4 & 38.8 \\
\hline uth & 9.1 & 58.8 & 59.4 & 0.5 & 30.2 & 30.8 & 0.8 & 50.4 & 51.3 & 0.3 & 39.8 & 40.8 & 5.7 & 65.3 & 66.0 & 4.4 & 24.0 & 24.8 & 52.8 & 52.4 & 53.2 & 5.1 & 4.6 & 35.7 \\
\hline lest & 62.4 & 62.1 & 62.8 & 27.5 & 27.2 & 27.8 & 56.7 & 56.1 & 57.2 & 31.3 & 30.5 & 32.1 & 66.8 & 66.3 & 67.3 & 24.2 & 23.7 & 24.6 & 58.4 & 58.0 & 58.9 & 30.7 & 30.2 & 31.3 \\
\hline 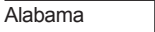 & 2.6 & 1.2 & 64.0 & 1.9 & 30.8 & 33.1 & 55.7 & 4 & 57.4 & 39.6 & 37. & 1.5 & 9.8 & 8.4 & 71.2 & 4.9 & 23.3 & 26.5 & 56.8 & . & 59.1 & 36.5 & $\cdots$ & 8.9 \\
\hline aska & 3.3 & 3.6 & 69.0 & 5.6 & 3.1 & 28.1 & 9.1 & 45.5 & 52.6 & 4.1 & 6 & 7.6 & 3.9 & 7.2 & 80.5 & 2.1 & 16.7 & 27.4 & 71.9 & 67.1 & 76.7 & 2.3 & .2 & 6.4 \\
\hline $\mathrm{zC}$ & 5.5 & 55.3 & 57.7 & 9.1 & 27.8 & 30.3 & 47.4 & 44.4 & 50.3 & 35.2 & 32.0 & 38.5 & 3.9 & 2.2 & 65.6 & 3.9 & 22.1 & 25.7 & 51.3 & 49.7 & 53.0 & 32.7 & 1.0 & 4.4 \\
\hline & 9.5 & 7.9 & 51.1 & 5.1 & 3.8 & 46.4 & .1 & 2.4 & 5.9 & 1.1 & 9.4 & 2.7 & 7.9 & 5.2 & 60.6 & 9.0 & 36.5 & 41.4 & 46.8 & 44.3 & 49.4 & 4.4 & & 17.0 \\
\hline alifornia & 59.9 & 59.4 & 60.4 & 1.0 & 30.6 & 31.5 & 50.0 & 47.4 & 52.6 & 37.4 & 34.6 & 40.1 & 2.8 & 62.1 & 63.5 & 28.7 & 27.9 & 29.4 & 57.1 & 56.5 & 57.8 & 3.3 & 0 & 34.1 \\
\hline & 8.0 & 6.8 & 69.1 & 9.0 & 17.9 & 20.1 & 56.4 & 4.5 & 58.2 & 23.2 & 20 & 25.7 & 7.0 & 75.6 & 78.4 & 3.2 & 11.9 & 14.6 & 59.3 & 56.9 & 61.8 & 25.5 & & 27.4 \\
\hline & 5.0 & 3.5 & 76.6 & 2.0 & 06 & 23.4 & 5.3 & 73.9 & 76.7 & 3.5 & 1 & 7.3 & 2.0 & .6 & 83.4 & 5.7 & .0 & 17.3 & 58.8 & .1 & 62.5 & 6.3 & & 9.9 \\
\hline Fid & 0.5 & 67.1 & 73.8 & 5.1 & 22.4 & 27.9 & 56.4 & 52.3 & 60.4 & 36.6 & 31 & 41.4 & 6.3 & 73.2 & 79.4 & 19.8 & 16.8 & 22.8 & 59.7 & 50.3 & 69.1 & 37.9 & 29.3 & 46.4 \\
\hline & 5.5 & 1.5 & 59.6 & 3.7 & 9.5 & 48.0 & $\mathrm{~N} / \mathrm{A}$ & N/A & $\mathrm{N} / \mathrm{A}$ & N/A & N/A & I/A & I/A & /A & N/A & I/A & N/A & N/A & 5.5 & & 59.6 & 3.7 & & 8.0 \\
\hline brid & 58.4 & 7.6 & 59.2 & 5.5 & 24.8 & 26.1 & 45.9 & 43.5 & 48.4 & 35.8 & 32.6 & 39.0 & 0.2 & 9.2 & 61.1 & 23.7 & 22.9 & 24.5 & 56.2 & 54.7 & 57.6 & 28.2 & 6.8 & 29.6 \\
\hline & 58.6 & 57.8 & 59.4 & 2.0 & 31.2 & 32.7 & 48.4 & 47.1 & 49.7 & 42.8 & 40 & 44.8 & 3.7 & 62.8 & 64.7 & 26.8 & 25.9 & 27.7 & 48.0 & 46.2 & 49.8 & 42.1 & 39.5 & 44.7 \\
\hline & .9 & 5.8 & 79.9 & 0.9 & 8.8 & 22.9 & 9.1 & & 0.3 & 5.9 & 21 & .1 & .1 & .5 & 82.8 & 9.7 & 16 & 2.8 & .8 & 8 & 8.7 & 6.7 & & 1.5 \\
\hline 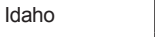 & 64.6 & 62.7 & 66.5 & 4.7 & 23.1 & 26.3 & 58.4 & 56.0 & 60.7 & 29.1 & 26 & 31.9 & 0.9 & 7.8 & 74.0 & 19.4 & 16.6 & 22.2 & 63.9 & 60.9 & 66.8 & 26.4 & 8 & 30.0 \\
\hline (1) & 5.9 & 65.2 & 66.6 & 0.8 & 30.2 & 31.4 & 2.8 & 62.0 & 63.5 & 36.6 & 34.9 & 38.4 & 3.9 & 3.1 & 74.8 & 23.2 & 22.5 & 23.9 & 53.8 & 52.1 & 55.5 & 1.4 & 1 & 42.7 \\
\hline & .4 & 5.4 & 67.5 & 6.0 & . & 27.0 & 2.3 & 61.1 & 3.5 & 26.4 & 2 & 28.4 & 6.3 & 4.9 & 77.6 & 8.4 & 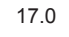 & 19.7 & 54.6 & 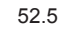 & 56.7 & 77.0 & & 39.0 \\
\hline 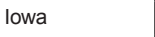 & 4.1 & 72.8 & 75.5 & 4.5 & 23.2 & 25.8 & 71.9 & 71.9 & 72.0 & 27.1 & 25 & 28.8 & 5.9 & 4.4 & 87.4 & 13.6 & 11 & 15.6 & 65.3 & 62.2 & 68.5 & 31.7 & & 35.0 \\
\hline & .4 & 0.1 & 72.8 & 2.4 & 1.1 & 3.7 & 7.6 & 7.0 & 3.2 & 4.9 & 22 & 8.0 & 1 & 6 & 6 & .1 & 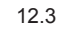 & 5.9 & .5 & & & 8.4 & & 0.7 \\
\hline & 1.8 & 0.5 & 63.0 & 3.6 & 32.2 & 34.9 & 1.1 & 49 & 52.6 & 43.2 & 4 & 45.1 & 6 & 5 & 73.7 & 24.3 & 22 & 26.0 & 65.1 & 62.3 & 68.0 & 31.2 & & 4.2 \\
\hline & 1.3 & 0.1 & 52.6 & 4.1 & 2.8 & 45.3 & 4.6 & 42.9 & 46.2 & 48.7 & 46.6 & 50.8 & 8.5 & 6.7 & 60.3 & 6.8 & 35.3 & 38.3 & 46.4 & 44.1 & 48.6 & 1.2 & 4 & 54.0 \\
\hline a & 1.4 & . & 66.6 & 2.5 & & 4.9 & & & 5 & 11.0 & & & & & 76.7 & 4.0 & re & 1.2 & 8.0 & & & 6.7 & & 44.2 \\
\hline & 4.1 & 3.0 & 75.3 & 2.8 & 1.8 & 23.7 & 70.3 & 69.5 & 71.1 & 26.6 & 22 & 30.8 & 8.6 & 7.5 & 79.8 & 18.3 & 17 & 19.4 & 55.0 & 52.2 & 57.7 & 41.7 & .8 & 44.6 \\
\hline & 7.0 & 6.0 & 78.0 & 4.0 & 3.2 & 24.9 & $\mathrm{~N} / \mathrm{A}$ & A & N/A & $\mathrm{N} / \mathrm{A}$ & & N/A & .3 & .3 & 82.4 & 9.4 & 18.4 & 0.4 & 2.4 & .3 & .5 & 9.8 & & 2.2 \\
\hline & 67.7 & 6.9 & 68.4 & 0.9 & 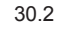 & 31.6 & 4.8 & & 5.4 & 36.5 & & & & & 77.0 & 2.3 & 2 & 3.4 & 52.3 & 50.6 & 53.9 & 5.3 & & 46.8 \\
\hline & 77.9 & 77.0 & 78.8 & 18.2 & 17.4 & 19.0 & 72.4 & 72.1 & 72.6 & 22.6 & 21.3 & 23.9 & 3.4 & 82.5 & 84.2 & 13.5 & 12.3 & 14.7 & 70.7 & 69.1 & 72.4 & 24.6 & .2 & 27.1 \\
\hline & 9.3 & 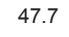 & 50.9 & 1.0 & 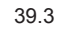 & & & & 4.9 & .1 & & & 3.2 & & 65.9 & 8.7 & 25 & 1.5 & 7.7 & & 42.1 & 8.0 & & 3.2 \\
\hline & 6.7 & 5.7 & 67.6 & 8.6 & 7.6 & 29.6 & 56.3 & 54 & 57.6 & 38.0 & 36 & 39.8 & 5.4 & 1 & 76.7 & 20.4 & 19 & 21.6 & 56.4 & 53.8 & 59.0 & 38.7 & & 1.4 \\
\hline & 5.7 & 2.7 & 68.6 & 1.9 & 9.6 & 24.2 & 1.3 & .5 & 3.1 & 3.1 & 20 & .8 & 7.5 & 2.7 & 82.2 & 8.8 & 11.9 & 5.6 & 1.2 & 8 & 5.7 & 0.1 & 3 & 6.0 \\
\hline & 1.2 & & & 1.2 & & & 70.0 & & & 23.7 & & & & & & & & & 0.6 & & & 5.0 & & 8.2 \\
\hline & 7.4 & 65.6 & 69.2 & 14.4 & 12.9 & 15.9 & 66.5 & 63.6 & 69.4 & 16.6 & 12.0 & 21.2 & 9.3 & 67.7 & 71.0 & 14.7 & 12.2 & 17.1 & 65.4 & 63.4 & 67.5 & 14.1 & 11.7 & 16.5 \\
\hline & 7.2 & 5.4 & 79.0 & 0.2 & م & 22.1 & 72.2 & 71.4 & 3.0 & 23.3 & 19 & 27.0 & 3.4 & & 86.7 & 4.5 & 12 & 6.9 & 7.3 & .1 & .5 & 2.0 & & 7.5 \\
\hline & 4.0 & 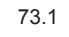 & 74.8 & 0.3 & 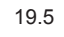 & & I/A & & $\mathrm{N} / \mathrm{A}$ & N/A & & & 7.0 & & 77.8 & 17.5 & & & 49.8 & & & 42.0 & & 5.5 \\
\hline ing & 7.4 & 5.4 & 49.4 & 2.4 & 0.4 & 4.3 & 7.9 & 3 & 0.5 & 53.7 & 51.3 & .1 & 9.5 & 6.2 & 52.9 & 8.7 & .6 & 1.8 & 5.0 & 52.2 & .8 & 4.4 & 3 & 7.4 \\
\hline & 6.4 & 0. & . & & & & & & & .2 & & & & & & & & & 1.0 & & & 4.0 & & 0.0 \\
\hline & 0.2 & 9.2 & 61.1 & 2.2 & 1.3 & 33.1 & 2.5 & 51.4 & 3.5 & 40.5 & 39.2 & 1.8 & 5.4 & 3.9 & 66.9 & 26.7 & 25.2 & 28.3 & 60.2 & 58.7 & 61.8 & 1.9 & 3 & 3.5 \\
\hline & 5.7 & 3.1 & 78.3 & 9.0 & 0.5 & 21.5 & 2.9 & 71.8 & 4.1 & 1.9 & 9. & 4.8 & 2.0 & 16.8 & 87.2 & 13.6 & & 18.9 & 76.0 & 70.8 & .3 & 17.6 & .5 & 1.7 \\
\hline & .5 & 8.8 & 70.2 & 6.2 & & & & & 6.0 & 29.8 & & & & & & & & & .2 & & & 4.2 & & .3 \\
\hline Oklahoma & 5.1 & 3.8 & 56.5 & 4.8 & 3.5 & 6.2 & 8.4 & 46.7 & 50.0 & 40.3 & 8.6 & .1 & 3.2 & 1.1 & 5.2 & 8.3 & 25.8 & 0.7 & 3.1 & .8 & .5 & 6.5 & 4 & 9.6 \\
\hline & & 0.0 & 69.5 & 1.0 & 0. & 2.7 & 9.7 & 00.1 & 1.3 & 26.7 & & & 0.2 & & & 18.1 & & & 70.1 & 08.3 & 71.8 & 0.6 & .4 & 22.7 \\
\hline & 0.5 & 9.8 & 71.2 & 7.5 & 26.8 & 28.1 & 65.8 & 65.3 & 66.3 & 31.9 & 30.4 & 33.4 & 8.7 & 78.0 & 79.4 & 19.7 & 19.1 & 20.4 & 50.0 & 48.0 & 52.0 & 16.8 & .9 & 48.8 \\
\hline & 2.9 & 0.7 & 75.1 & 5.9 & 3.6 & 28.3 & $\mathrm{~N} / \mathrm{A}$ & N/A & N/A & N/A & N/A & & 8.9 & 7.2 & 80.7 & 20.8 & 18.1 & 3.6 & 60.0 & 55.4 & 64.6 & 6.7 & 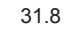 & 1.6 \\
\hline & 0.4 & 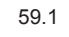 & 817 & 9.3 & (2) & & 1.8 & 52.9 & 7 & 8 & 32. & & 3 & & & 1 & & & .5 & & .4 & 0.0 & & 3.7 \\
\hline th Dakota & 7.7 & 5.1 & 70.2 & 9.4 & 26.2 & 2.6 & 2.1 & 60.4 & 3.9 & 1.3 & 27.7 & .9 & 1.4 & 1 & 4.7 & 9.3 & 6 & .9 & 7.2 & .4 & .1 & 4.3 & 3 & 2.3 \\
\hline & 3.7 & 2.0 & 64.8 & 1.6 & 0.0 & 0.0 & 57.1 & 00. & 58.5 & 39.0 & 3 & & 3 & 11.0 & 10.1 & 22.7 & 21.5 & 24.1 & 57.3 & 55.3 & 59.3 & 36.5 & 4 & 38.5 \\
\hline & 3.4 & 2.9 & 54.0 & 0.3 & 29.8 & 30.8 & 49.1 & 47. & 50.5 & 33.7 & 32.5 & & 1.6 & 60.9 & 62.2 & 24.2 & 23.4 & 25.0 & 46.7 & 45.9 & 47.5 & 5.4 & & 6.3 \\
\hline & 4.7 & 73.2 & 76.2 & 13.8 & 2.5 & 15.1 & 8.5 & 66.6 & 0.4 & 21.0 & 17.8 & .2 & 9.1 & 7.6 & 80.6 & 11.0 & 5 & 2.5 & 62.4 & 58.8 & 66.0 & 9.7 & 3 & 3.1 \\
\hline & 4 & 0.9 & 65.9 & 7.9 & & 40.5 & 9.3 & 57.2 & 1.3 & 41.6 & & & 2.1 & 01.0 & 77.3 & 29.6 & 24.6 & & N/A & N/A & N/A & V/A & N/A & N/A \\
\hline & 5.1 & 4.3 & 75.9 & 19.3 & 18.5 & 20.1 & 8.1 & 62.7 & 65.5 & 30.6 & 28.3 & 32.9 & 79.6 & 78.6 & 80.6 & 14.6 & 13.6 & & 69.8 & 68.0 & 71.6 & 4.9 & 3.2 & 6.6 \\
\hline & 7.9 & 66.9 & 68.9 & 6.5 & 25.6 & 27.4 & 59.3 & 57.6 & 61.0 & 38.0 & 35.9 & 40.2 & 72.3 & & 73.6 & 21.6 & 20.4 & 22.7 & 61.9 & 59.9 & 63.9 & 32.4 & 30.2 & 34.6 \\
\hline & & 7.5 & 1.8 & 7.9 & $\sigma^{2}$ & 40.1 & .0 & 50 & 5.1 & 43.8 & 41 & 46.6 & 8.1 & 3 & 70.8 & 30.9 & 27.8 & .9 & 53.0 & 47.6 & 58.4 & 2.1 & .9 & 9.2 \\
\hline & 5.0 & 74.1 & 75.9 & 22.9 & 2.0 & 23.8 & 70.5 & 70.3 & 0.7 & 25.3 & 24.0 & 26.6 & 84.6 & 83.6 & 85.6 & 14.9 & 13.7 & 16.2 & 63.0 & 60.4 & 5.7 & 34.1 & 2.0 & 6.2 \\
\hline & & & $t$ & 5.0 & & & 70.4 & & 72.3 & 23.6 & & & N/A & & N/A & N/A & $\mathrm{N}$ & & 72.6 & 68.0 & 77.2 & 27.0 & 20.7 & 33.3 \\
\hline uento & 4.5 & 43.2 & 5.1 & 1.1 & 9.9 & 2.3 & 5.1 & 28.4 & 1.8 & 62.8 & 0.5 & .1 & 5.7 & 4.2 & 47.3 & 50.2 & 8.8 & 51.6 & 43.7 & 41.4 & 46.0 & 50.3 & 1 & 3.4 \\
\hline
\end{tabular}

\section{$\mathrm{N} / \mathrm{A}=$ Not applicable}

'Levels of urbanization are defined as follows: rural consists of ACS geographic components "Not in metropolitan or micropolitan statistical area" and "In micropolitan statistical area";

suburban includes "In metropolitan statistical area- not in principal city" and central city includes "In metropolitan statistical area- in principal city".

2Data are based on 2008 American Community Survey estimates.

Source: American Community Survey 


\section{E N D N O T E S}

1. Overlap in public and private health insurance coverage is due to both individuals with double coverage and survey error, but there is no way to differentiate the two, according to officials at the U.S. Census Bureau. However, the rate of overlap is small (just over 2 percent of children in the United States).

2. Note that this does not include places where data are unavailable for rural places, suburban places, or central cities. 3. http://factfinder.census.gov/home/saff/main.html?_ lang=en\&_ts.

4. To access the Accuracy of the Data document, visit http://www.census.gov/acs/www/UseData/.

5. To calculate the confidence intervals for the estimates, refer to the American Factfinder tool and the Accuracy of the Data documentation.

\section{ABOUT THE AUTHORS}

Beth Mattingly is director of research on vulnerable families at the Carsey Institute and a research assistant professor of sociology at the University of New Hampshire. Her research addresses child poverty and its implications, the consequences of family violence; the intersections of gender, work, and family; and family well-being (beth.mattingly@unh.edu).

Michelle Stransky is a research assistant at the Carsey Institute and a doctoral candidate in the Department of Sociology at the University of New Hampshire (mlu6@cisunix.unh.edu).

\section{A C K N O W L E D G M EN T S}

We are grateful for feedback from Mil Duncan, Curt Grimm, Terri Rippett, and Sally Ward at the Carsey Institute and Debbie Stein at the Hatcher Group.

\section{$\triangle$ UNIVERSITY Ei of NEW HAMPSHIRE}

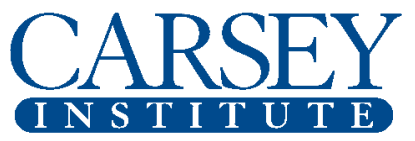

Building knowledge for families and communities

The Carsey Institute conducts policy research on vulnerable children, youth, and families and on sustainable community development. We give policy makers and practitioners timely, independent resources to effect change in their communities.

This work was supported by the Annie E. Casey Foundation's initiative to strengthen rural families, the W. K. Kellogg Foundation, and an anonymous donor.

Huddleston Hall

73 Main Street

Durham, NH 03824

(603) 862-2821

www.carseyinstitute.unh.edu 